CAHIERS DE

NARRATOLOGIE

\section{Cahiers de Narratologie}

Analyse et théorie narratives

$30 \mid 2016$

Street Art 2

\title{
Penser le roman comme un dispositif narratif. Vers une subjectivation du lecteur
}

\author{
Hélène Crombet
}

\section{(2) OpenEdition}

\section{Journals}

\section{Electronic version}

URL: http://journals.openedition.org/narratologie/7535

DOI: 10.4000/narratologie.7535

ISSN: $1765-307 X$

\section{Publisher}

LIRCES

\section{Electronic reference}

Hélène Crombet, "Penser le roman comme un dispositif narratif. Vers une subjectivation du lecteur », Cahiers de Narratologie [Online], 30 | 2016, Online since 28 July 2016, connection on 01 May 2019. URL : http://journals.openedition.org/narratologie/7535; DOI : 10.4000/narratologie.7535

This text was automatically generated on 1 May 2019.

Article L.111-1 du Code de la propriété intellectuelle. 


\title{
Penser le roman comme un dispositif narratif. Vers une subjectivation du lecteur
}

\author{
Hélène Crombet
}

\section{Introduction : l'expérience autoréflexive du roman comme dispositif de lecture}

1 Certaines façons de lire peuvent soulever des manières d'être : " [l]a lecture est d'abord une "occasion" d'individuation", écrit Marielle Macé1. Les enjeux liés à l'expérience de la lecture sont effectivement susceptibles d'interroger une "perspective du sujet $»^{2}$ : comment le lecteur travaille-t-il et traverse-t-il le roman et comment, dans un mouvement réciproque, le roman le travaille-t-il et le traverse-t-il ?

2 A partir de la thèse que nous avons réalisée, nous avons choisi de nous focaliser sur des romans de la littérature française du $\mathrm{XXI}^{e}$ siècle qui ont en commun de relater la guerre d'Algérie, à la faveur d'une mise en récit singulière: le lecteur de ces romans est effectivement invité à se projeter dans la pensée d'une pluralité de personnages qui lui livrent leur point de vue respectif au sujet de cette période historique; parmi eux, plusieurs font montre d'une noirceur particulière.

3 La spécificité de ces mises en récit nous a amenée à nous interroger sur les enjeux de la lecture qu'elles suggèrent, à travers un phénomène de déstabilisation des frontières liminaires de l'identité du sujet. Dans quelle mesure ces procédés narratifs constituent-ils des mécanismes de projection du lecteur? Ce processus de dessaisissement apparaît d'autant plus brûlant qu'il est amené à partager la focalisation de personnages de fiction révélant une cruauté manifeste dont il s'agit de déterminer les propriétés : comment se caractérise cette logique de pensée qui les anime? Mais il faudrait aussi s'interroger sur la dimension unilatérale de cette lecture : en entrant dans l'intériorité psychique de telles figures, le lecteur demeurerait-il dans une seule posture de fascination subjuguée qui le 
désintriquerait inexorablement de lui-même ? Ne reprendrait-il pas récursivement ses esprits?

4 Dans la perspective de saisir les enjeux liés à cette expérience d'oscillation identitaire, nous envisagerons le rapport du lecteur au roman conçu comme un dispositif narratif, à l'aune d'une approche pragmatique et interactionniste de la situation lectorielle tendant à suspendre temporairement l'incrédulité du sujet ${ }^{3}$. Nous entendrons dans sa dimension autoréflexive le terme de "dispositif ", qui lui permet de faire l'expérience d'un entredeux : il suggère effectivement une relation de l'individu au monde marquée par une interaction ambivalente, parce qu'il «touche à la constitution de l'identité [...], établit une médiation affective et corporelle entre soi-même et le monde, entre soi-même et autrui, et finalement entre soi et soi $»^{4}$. Dans l'acception que nous lui conférons, le dispositif est fondamentalement anthropomorphique: il se compose effectivement de personnages de fiction entendus comme des supports de projection du sujet, qui lui permettent de faire l'expérience de sa propre identité en le faisant devenir autre pour un temps donné.

5 A travers notre article, nous analyserons ces techniques et procédés narratifs qui concourent à projeter le lecteur dans la pensée de personnages de fiction, à partir d'extraits de romans récents relatant la guerre d'Algérie. Nous ferons ensuite émerger les enjeux relatifs à deux expériences d'altération identitaire liées, précisément, au degré d'altération qu'elles suscitent : la première sera caractérisée par une forme d'altération relative, dans la rencontre du lecteur avec une simple "inquiétante étrangeté »; la seconde, comme une expérience d'altération radicale, le lecteur se projetant dans la pensée de personnages de fiction sadiques dont il est amené à partager la focalisation. Cette dernière nous permettra notamment de faire émerger le caractère polémique de l'abjection entre sentiment de fascination et sentiment de révulsion, induisant un phénomène de vacillation identitaire qui ébranle catégoriquement les frontières de son identité. Nous ferons alors émerger une perspective ontologique induite par cette expérience singulière de la lecture de romans entendus comme des dispositifs, qui offrent au sujet la possibilité d'un processus d'individuation autoréflexif. Cette perspective, dynamique, devrait effectivement être considérée à l'aune d'un principe de subjectivation dual, qui fait alterner des moments de battements pendulaires entre dépossession et repossession de soi.

\section{Extraits de romans : des dispositifs immersifs}

6 Trois extraits de romans illustreront notre propos. Le premier est issu de Même pour ne pas vaincre de Stéphane Chaumet, publié en 2011. Il s'agit de l'incipit de ce roman "polyoptique ", dans lequel l'un des personnages témoigne de sa participation à la guerre d'Algérie :

Assis par terre, la saignée des bras sur les genoux, bras ballants, la bière par terre au bout du pied, le front humide, la casquette relevée en arrière dégageant le front essuyé d'un revers de manches, préférant baisser les yeux sur la terre craquelée assoiffée, la terre qui donne soif, que les garder sur la beauté étouffante de l'horizon menaçant, cette saccade de montagnes tout en plis où la mort couve ses œufs, où il faudra retourner avec l'angoisse que l'œuf cette fois vienne éclore pour soi, juste pour soi dans une terrible offrande du hasard qui ne touchera pas l'autre corps à quelques centimètres du sien, autant baisser les yeux sur cette terre toute sèche, poussiéreuse, terne, avec des mottes comme de petits cailloux, et d'une 
pichenette ou prenant l'une d'elles dans la main, à peine serrant, elle s'émiette, comme le temps, notre temps ici n'est que ces petites concrétions de terre sèche misérable, fade, qui soudain s'émiette, se pulvérise, plus rien qu'une trace poussiéreuse insignifiante sur les doigts secs eux aussi [...]. ${ }^{6}$

7 Dans cet incipit, qui suscite une sensation d'étouffement, le personnage relate le quotidien angoissant de son service. Quelques marques de ponctuation viennent scander la pensée du personnage, dans l'emportement spasmodique et heurté d'une pensée asyntaxique qui semble faire irruption dans le monde fictif du roman, sans être délimitée dans un cadre spatio-temporel bien régulé, le temps - à l'image de la terre - paraissant se pulvériser, s'émietter dans une cascade de paroles déchaînées.

Le deuxième extrait est tiré de C'était notre terre de Mathieu Belezi, publié en 2008. Roman également " polyoptique ", C'était notre terre met en scène six personnages de fiction, dont cinq appartiennent à une même famille de colons en Algérie. L'extrait suivant relate des exécutions sommaires, commises par le père de famille, membre de l'OAS :

- Pas un habitant ne doit rester en vie! Pas un!

C'est le mot d'ordre qu'on se passe d'une maison à l'autre, que pas un homme, pas une femme, pas même un chien ne survive, dans la chaleur des flammes qui montent jusqu'aux étoiles on n'entend plus les cris, on ne voit plus les bouches qui se tordent, les yeux qui se ferment, les ballons de baudruche des poitrines qui se dégonflent par les trous que leur font les balles [...] et on patauge dans le sang, dans ce sang assassin qui ne nous veut que du mal, on en est éclaboussé, trempé jusqu'aux os, il nous soûle à la façon d'un mauvais vin, il nous empoisonne l'estomac, il nous enlise dans les corps qui jonchent les ruelles [...]

Enrouée ma voix se perd dans le staccato de ma dernière rafale de mitraillette, je n'ai plus de munitions, je tombe à genoux, roule sur le côté, tente d'appuyer mon dos contre le mur d'un gourbi qui n'a pas brûlé [...]

Moreno m'aide à me relever, ensemble et à travers les derniers feux nous traversons le champ de bataille, au ras du sol des flammes lèchent les mollets, des corps qui n'en finissent pas de mourir gémissent malgré eux [...]. ${ }^{7}$

Dans cet extrait déferle tout le délire de destruction du personnage enivré par le caractère quantitatif des actes meurtriers qu'il commet, à travers le déchaînement extatique d'une frénésie accumulant les victimes, dans la volonté assourdissante des armes de les réduire au silence et à l'anéantissement.

Le troisième extrait est issu du roman Des hommes, de Laurent Mauvignier, paru en 2009.

Ce fragment relate l'attaque profondément brutale d'un village algérien, du point de vue de l'un des soldats :

Des enfants crient devant la porte d'une maison. Ils restent immobiles, deux petits, debout, ils crient jusqu'à ce qu'une femme vienne les chercher et les emmener avec elle s'asseoir sur la place, serrés, tous, les voisins, les amis, les autres, la famille, tous, pourvu qu'ils soient femmes, vieux, enfants, tous blottis les uns contre les autres à hauteur des jambes des soldats, avec la pointe des canons qui danse devant leurs yeux et la poussière étouffante et chaude, épaisse, blanche et qui brouille les yeux et les odeurs et donne un goût sec et farineux dans la bouche [...], on entend [...] les portes qu'on fracasse, des cris de femmes, quelques femmes enfermées ou cachées des femmes jeunes avec des couleurs vives, les tissus rouge, bleu, jaune, elles résistent, il les faut les pousser, il faut qu'on les pousse avec les pointes des armes $\left[\ldots . . .{ }^{8}\right.$

11 La narration des pillages et des mises à sac dans le village algérien se déploie ainsi à travers une logorrhée insatiable de cruauté, relatant avec force détails les violences commises. La syntaxe, profondément démise, semble faire écho à la fureur du personnage 
déchaîné, qui s'opère de manière frénétique. Est en outre soulignée l'accumulation des victimes retranchées au pied des soldats qui les tiennent en leur possession, omnipotents.

\section{La projection du lecteur dans la pensée des personnages : techniques et procédés narratifs}

Dans une approche "post-classique » de la narratologie", nous analyserons plusieurs techniques et procédés narratifs qui tendent, dans ces extraits, à projeter le lecteur dans la pensée des personnages de fiction.

D'une part, le lecteur accède au monde du texte à travers la focalisation ${ }^{10}$ prise en charge par le personnage de fiction. Le "focalisé $~^{11}$ est ainsi livré au lecteur à partir de sa perspective focale, le « je-narrant » se confondant avec le " je-narré » ${ }^{12}$. Par conséquent, le lecteur accède au monde du texte par le biais de champs de focalisation restreints ${ }^{13}$. Ce mode d'accès à l'histoire semble projeter plus intensément le lecteur dans la pensée des personnages de fiction, puisqu'il est amené à partager leur point de vue. L'on peut toutefois remarquer l'emploi du pronom « on » dans les deux derniers extraits que nous avons cités, tout se passant comme si se produisait un phénomène d'indiscernabilité des membres de l'OAS dans C'était notre terre et des soldats dans Des hommes, plongés dans un état d'ivresse extatique qui entraîne un flottement des frontières de leur identité.

D'autre part, le récit dans ces romans est pris en charge par une pluralité de personnages comme autant de perspectives consonantes ou dissonantes sur la guerre d'Algérie. Le caractère disjonctif de cette forme narrative peut susciter un phénomène de manège vertigineux pour le lecteur, soumis à des décrochages réguliers de focalisations ${ }^{14}$. En effet le récit, dans Même pour ne pas vaincre, est pris en charge par des personnages dont la focalisation fonctionne en résonance et en dissonance sur la guerre d'Algérie: outre le point de vue de Philippe Bougeard dont nous avons prélevé un extrait, les monologues de son neveu, Jean, ponctuent l'ensemble du roman, entrecoupant le récit de deux autres personnages, Karim et Sania. Le roman-fleuve C'était notre terre est quant à lui porté, depuis la France et l'Algérie, par la narration de six personnages comme autant de perspectives différentes sur cette période de l'Histoire : celui du personnage, père d'une famille de colons, des quatre autres membres qui la composent et de leur gouvernante, d'origine berbère ; l'ossature narrative de Des hommes s'articule autour de quatre chapitres qui livrent le point de vue d'une pluralité d'anciens soldats appelés à faire leur service militaire lors de la guerre d'Algérie.

Amené à partager la focalisation prise en charge par une multitude de personnages, le lecteur peut donc être soumis à l'expérience d'une déliaison étourdissante et assourdissante. Néanmoins, la dimension vocale ne saurait être mise de côté : dans les passages cités, le lecteur a affaire au genre du monologue intérieur ${ }^{15}$; il est effectivement confronté au type du «monologue remémoratif» dans le premier extrait, et au «monologue autonome» dans les deux derniers extraits. Ces deux types de monologues créent l'illusion d'un déroulement ininterrompu de la psyché, qui semble jaillir avant toute organisation par la pensée logique. Haletant, le rythme de ces extraits se caractérise en outre par une scansion et une ponctuation marquées par leur aspect haché, traduisant l'idée d'une suffocation. 
16 De plus, le langage employé est un langage courant, ce qui peut induire un sentiment de proximité plus forte entre le lecteur et le personnage, qui ne parle pas «comme dans un livre».

17 Enfin, dans le premier extrait, le lecteur est confronté à un « incipit in medias cogitationes » ${ }^{16}$. C'est-à-dire que nous, lecteur, sommes amené à épouser le point de vue focal du personnage de fiction, dès lors que nous entrons dans l'univers textuel du roman : de manière immédiate, nous sommes projeté dans ses pensées, faisant ainsi l'expérience $d^{\prime}$ ' histoires potentielles ou inchoatives » à l'état d'esquisses ${ }^{17}$. Tout se passe ainsi comme si le lecteur voyait défiler des pensées en train de se faire, dans l'émergence d'une parole paraissant faire irruption dans le roman; roman qui semble mettre un terme et des termes à cette parole, en lui donnant simultanément une présence et une absence.

\section{Une expérience d'altération relative : le télescopage extatique du lecteur}

18 L'ensemble de ces techniques et de ces procédés narratifs concourt à immerger le destinataire ${ }^{18}$ dans le point de vue focal des personnages de fiction, et ce de manière particulièrement vertigineuse, frontale et brutale. Ces mécanismes d'immersion peuvent entraîner un processus d'incarnation du lecteur, plongé dans l'intériorité des personnages ; personnages de fiction dont il ne faudrait évidemment pas sous-estimer la puissance, en termes de vecteurs émotionnels ${ }^{19}$. Ils sont ainsi susceptibles de constituer des supports privilégiés de l'identification du lecteur. Ce dernier peut être saisi, dessaisi de lui-même, en faisant l'expérience d'un processus d' « hallucination paradoxale ${ }^{20}$ : il serait submergé par des "impulsions sensibles", telles des perceptions externes provenant de l'extérieur de l'appareil psychique, pour atteindre précisément l'intériorité de sa psyché. Ces impulsions sensibles sont en mesure de prendre une "voie régrédiente » en amoindrissant les facultés critiques du sujet, soumis à un état proche de la «rêverie ». Il peut effectivement être déstabilisé par cette irruption dans de tels monologues l'un remémoratif, les deux autres autonomes qui le soumettent au joug d'une certaine curiosité, d'une certaine envie de voir.

19 Le lecteur est ainsi susceptible de faire l'expérience d'une forme de fascination subjuguée dans sa lecture, soumis à un phénomène de médusation ${ }^{21}$ qui le pétrifie, qui le ravit et qui le fait se raviser en lui-même. Tout se passe comme si cette modélisation de psychés tout en turbulences se mettait en coïncidence avec son propre musement ${ }^{22}$ intérieur, telle une narration "naturelle $»^{23}$, c'est-à-dire spontanée, évoquant l'expérience du monde parfois chaotique de la conscience humaine. Il peut ainsi être surpris, dépris de lui-même, par cet accès qui lui est donné à la verbalisation directe de ce qui est pensé par les personnages. Dans le premier extrait, ce télescopage s'opère de manière d'autant plus saisissante qu'il est amené à partager la focalisation du personnage de fiction dès les premières lignes du roman ; dans les deux autres, il peut être emporté par cette intrusion dans l'intériorité d'une pensée évoquant une violence extrême, qui s'effectue à travers une syntaxe tout aussi désaxée. 


\section{Une expérience d'altération radicale : l'épreuve polémique de l'abjection}

20 Un second degré d'altération identitaire peut être évoqué qui consiste en ce que, à travers les deux derniers extraits de romans que nous avons relevés, le lecteur est invité à épouser le point de vue de personnages qui font montre d'une très grande cruauté. Il est effectivement amené à partager la focalisation du membre de l'OAS participant aux massacres d'Algériens dans C'était notre terre, mais aussi à se projeter dans celle du soldat qui relate le saccage d'un village, dans le passage que nous avons extrait du roman Des Hommes. Quels sont alors les effets de l'irruption du lecteur dans la pensée de tels personnages?

Nous nous emploierons à présent à caractériser cette logique que nous pouvons qualifier de sadique. Celle-ci est régie par un processus double, paradoxal, qui tient d'une jubilation froide, faisant appel à deux éléments ${ }^{24}$ : un élément impersonnel d'une part, lié à des mécanismes de pensée pénétrés d'esprit mathématique et procédant d'un principe de pure Raison; élément impersonnel qui se conjugue à un élément personnel d'autre part, faisant appel à la singularité d'une toute-puissance surmoïque, et relevant d'un égoïsme absolu, en lien avec un profond délire, avec un déchaînement de la pensée.

De tels personnages procèdent à une énumération logique qui s'opère jusqu'à l'épuisement interminable, les scènes de férocité succédant aux scènes de férocité, à travers une itération systématique qui additionne les victimes. Dans le deuxième extrait, le caractère frénétique des massacres perpétrés par les membres de l'OAS apparaît à travers la désarticulation de la syntaxe, qui rend compte de la frénésie meurtrière du personnage plongé dans l'ivresse d'une extrême violence. La mention des « corps » à ses pieds, marquée par une forme de neutralité, témoigne de l'incapacité qui est la sienne à faire montre de différenciation vis-à-vis d'une autre vie humaine. Cette répétition meurtrière se déchaîne ainsi, indépendante de tout plaisir préalable, à travers une forme de frénésie qui s'accomplit dans l'insensibilitée ${ }^{25}$ du sadisme. Le dernier passage que nous avons relevé fait émerger cette volonté d'annihiler tout ce qui se trouve sur leur passage, à travers l'accumulation des victimes «blotti[e]s les uns contre les autres à hauteur des jambes des soldats ", dans la réitération d'une fureur inassouvie qui tend à révéler cette logique omnipotente. La psyché se trouve ainsi aux prises avec une action déliée, au moyen d'une méthode implacable et mécanique. Le désir est ici effectivement mû par la seule soif d'une destruction totale, par le seul dessein d'atteindre la négation absolue. Idée de négation néanmoins impossible à atteindre, qui est la cause de cet accomplissement, réitéré à l'infini, de processus uniquement négatifs, comme processus partiels de destruction. Cette répétition s'opère à travers la seule spontanéité d'un présent absolu - renvoyant aux monologues que nous avons déclinés plus haut -, dans la pure frénésie du meurtre, dans le spasme cruel d'une volonté de réduire l'écart entre ces processus de destruction partiels, et l'Idée de négation pure.

C'est parce que le lecteur se projette dans de tels systèmes de pensée que la lecture peut être particulièrement dérangeante et déstabilisante. Elle amène effectivement le lecteur à faire l'expérience périlleuse d'une confrontation avec l'immonde: il est en mesure de devenir lui-même la surface de projection du personnage, à travers un phénomène d'interversion chiasmique ${ }^{26}$. Le livre semble alors se faire «objet transnarcissique ${ }^{27}$, et la page, une surface réfléchissante entre sujet et objet, tels un obstacle et simultanément 
un réceptacle de la lecture. Dans un processus d'« hallucination négative », tout se passe effectivement comme si le lecteur lui-même était proprement concerné par le personnage de fiction. Tout se passe comme si, comme « regardant regardé », il se voyait, « en seconde vue », et devait s'appréhender dans un rapport d'altérité radicale ; comme radicalement séparé de lui-même.

Dessaisi, à travers une lecture marquée par une oscillation des limites de son identité, le lecteur est ainsi invité à penser sa propre - ou son impropre - complexité. Mais que peut-on dire alors de cette part d'opacité radicale qu'il découvre en lui-même?

\section{L'émergence d'une perspective ontologique fondamentalement oscillante}

Le regard du sujet braconnerait ${ }^{28}$ ainsi les lignes comme autant de logorrhées, de constructions verbales qui roulent sur le vide, et qui sont en prise directe avec son propre musement intérieur. La confrontation avec une pensée qui s'emballe jusqu'au détraquement, cahotant et chaotique, ça agace, ça secoue, ça violente ; quand tout à coup la brise de la pensée se brise ; quand tout à coup le familier se fait harcelant; quand le bruissement, toujours présent et absent, est interrompu et s'emballe simultanément, en devenant brisement.

Le sujet, prenant ainsi pour objet un tel musement déchaîné, qui paraît faire écho avec sa propre pensée en mouvement, s'y trouve subordonné, l'objet le prenant, l'interpellant, le convoquant. L'objet, c'est celui qui m'homologue infiniment, indéfiniment à lui, en ne me permettant pas d'être absolument détaché ou autonome ${ }^{29}$. L'objet, c'est celui qui s'oppose à mon désir, tout en même temps qu'il vient incessamment me manquer, me tirailler. Il se propose à mon désir, et simultanément vient s'objecter à ma volonté.

Que se passe-t-il, par conséquent, lorsque le sujet prend pour objet l'abject et devient le sujet de l'abject mais, du reste, qu'est-ce précisément que l'abject?

Le préfixe «ab-» latin renvoie étymologiquement à l'idée d'une séparation, d'un éloignement. Le terme " abject " provient du participe passé passif latin «abjectus », du verbe «abjicere », signifiant « jeter loin de soi », « rejeter au loin ». L'abject, c'est ce qui provoque notre dégoût, notre répulsion, notre aversion; c'est ce que nous ab-horrons; c'est ce qui nous fait horreur. L'abject m'aimante et me révulse, simultanément. Il convoque un autre en moi, aussi interdit que désiré, ébranlant la frontière entre sujet et objet, dans un phénomène d'indiscernabilité de la limite entre dedans et dehors, à la lisière entre mimicry et ilin $x^{30}$.

Une expérience de vacillation identitaire est ainsi articulée à travers l'interaction entre le sujet et ces dispositifs qui convoquent des contraires, entre sentiments de fascination et d'opprobre, entre pur et impur, entre propre et impropre. Le sujet apparaît tiraillé, tyrannisé à travers une lecture impulsive et répulsive; dans un écartèlement entre une tendance fascinée vers un double ${ }^{31}$ malveillant, et une prohibition, une renonciation soulevant l'idée de tabou comme " terreur sacrée "32, à travers une lecture comprise entre processus de régression, et sursauts de répression.

Toutefois, au moment où la projection du lecteur l'invite à s'égarer dans des territoires profondément instables, profondément déterritorialisants, aux confins de son identité (où la dimension spatiale nous apparait prééminente), en se prenant pour sujet du sentiment d' 
abjection qu'il ressent, l'on pourrait considérer que cette noirceur le protègerait, en lui permettant de ne pas s'annihiler à travers ce tréfonds déchainé. L'abject s'oppose à lui comme objet de son désir, qui pose la limite de son être comme vivant, en le protégeant de l'évanouissement.

31 Ainsi, à la faveur de ce sentiment d'abjection, le lecteur se ressaisit, la révulsion étant au fondement de l'émergence de sa conscience morale ${ }^{33}$, comme formation réactionnelle destinée à endiguer le flot, le flottement intérieur. Confronté à des moments de déprise de soi, le lecteur est effectivement amené à se reprendre, soumis à des sortes de piqûres convulsives et révulsives.

Ce phénomène de ressaisissement du lecteur apparait d'autant plus tangible que ces romans, comme "polyoptiques » ou multifocaux, lui proposent de considérer le récit à travers une texture stéréoscopique. La mise en récit est effectivement opérée à travers une convocation de singularités hétérogènes qui s'accordent, se nuancent, et parfois se contredisent réciproquement quant à leur vision de l'Histoire. Quoique ce " manège » des points de vue focaux puisse provoquer une sensation de vertige, la discontinuité narrative est également susceptible de favoriser un phénomène de distanciation du lecteur, car il doit lier en les lisant ces différentes visions qui lui sont proposées. Il doit ainsi faire l'effort de reconfigurer de lui-même l'agencement apparemment informel qui lui est livré, tout se passant comme si l'acte de reconfiguration de la mise en récit semblait advenir avant la mise en récit elle-même. L'aspect profondément éclaté de la narration, admettant des nuances et des antagonismes, n'offre effectivement pas de résolution conclusive au destinataire, invité à s'engager dans une lecture active qui l'amène à restructurer de lui-même le récit. Il doit ainsi s'appliquer à embrasser ces différentes perspectives focales, qui se déploient à l'aune d'un principe de ramification perpétuelle fonctionnant en résonnances et en dissonances dans la compréhension de logiques contraires.

\section{Conclusion : l'expérience du dispositif narratif, vers une subjectivation du lecteur}

Pensés comme des dispositifs, ces romans permettent au lecteur de faire l'expérience d'un entre-deux, dans un processus ambivalent entre deux pôles alternatifs, à travers une situation de battement entre deux mouvements contraires, répulsifs et impulsifs. Mis en présence du réel dans sa brutalité "obscène $~_{34}$, le lecteur est ainsi amené à faire une expérience frontale, qui ne peut le laisser indifférent. Confronté à de tels monologues telles des « histoires inchoatives $»^{35}$ qui l'introduisent instantanément dans la pensée de personnages de fiction sadiques, il est ainsi invité à faire l'épreuve de l'abject, suscitée par la mise en présence d'une violence extrême qui le regarde mais qui le garde simultanément de lui-même. Ce processus d'identification à des personnages dont il devient momentanément la surface de projection occasionne ainsi l'expérience d'une vacillation identitaire qui rend poreuses les frontières de son identité.

L'on pourrait penser que cette expérience renverrait à un seul phénomène de reconnaissance originaire, dans la répétition d'un processus de différenciation vis-à-vis d'un «pouvoir aussi sécurisant qu'étouffant $»^{36}$. Mais l'on pourrait également considérer ces romans comme des « dispositifs », tels des « espaces transitionnels » ou des « espaces intermédiaires " précisément occupés par des «sujets transitionnels » que 
constitueraient les personnages de fiction. Ce seraient des piliers fondamentaux, autour desquels s'opèreraient ces processus de différenciation et d'indifférenciation du sujet avec lui-même ${ }^{37}$.

Les personnages représenteraient dans cette perspective des matériaux d'élaboration de soi-même, à la faveur d'une traversée permanente dans le labyrinthe du multiple. Le sujet, à travers une lecture comprise comme un « branchement transversal $»^{38}$, se laisserait déterritorialiser, territorialiserait et déterritorialiserait lui-même d'autres territoires, d'autres mondes, d'autres modes d'être, dans l'épreuve périlleuse de la diversité de sa propre identité. Marielle Macé soulève ainsi la possibilité qui est donnée au lecteur d'expérimenter de la sorte une autre existence pour un temps donné : «L'expérience littéraire en tant que telle [...] se prolonge et se ressaisit [...] en pragmatique du rapport à soi et au monde sensible : en invitation à essayer de nouvelles dispositions cognitives, un autre corps, un autre rythme, un autre "soi" ». ${ }^{39}$

Ainsi, le personnage constituerait dans cette perspective le jalon catégoriel d'une construction identitaire de soi, qu'il s'agirait d'intégrer un peu, beaucoup, passionnément, voire, à la folie, dans une forme de (con)fusion momentanée; en se différenciant ainsi perpétuellement de soi-même, dans un travail d'autonomisation ${ }^{40}$ dynamique et permanent.

\section{NOTES}

1. Marielle Macé soulève effectivement la dimension autoréflexive de la lecture en ces termes : « devant les livres, nous sommes conduits en permanence à nous reconnaître, à nous "refigurer", c'est-à-dire à nous constituer en sujets et à nous réapproprier notre rapport à nous-mêmes dans un débat avec d'autres formes. » Voir Marielle Macé, Façons de lire, manières d'être, Paris, Gallimard « Nrf/essais », 2011, p. 18.

2. Francis Vanoye saisit la posture lectorielle telle « une rencontre entre un sujet et un texte, une personne et un morceau de langage ». Francis Vanoye, Récit écrit - récit filmique, Paris, Editions CEDIC, Coll. « Textes et non-textes », 1979, p. 26.

3. Ce « cadre communicationnel » tend à fournir des « instructions pragmatiques » au lecteur. Celles-ci sont vouées à suspendre les contraintes inhérentes à la « communication documentaire ", établie suivant un principe d' « isomorphisme » et de " dérivation ». Cf. Olivier Caïra, Définir la fiction. Du roman au jeu d'échecs, Paris, EHESS « En temps \& lieux », 2011, p. 79.

4. André Berten, « Dispositif, médiation, créativité : petite généalogie », Hermès, La Revue, 1999/3, n 25, p. 39.

5. Nous reviendrons sur l'emploi de ce terme dans la suite de notre propos.

6. Stéphane Chaumet, Même pour ne pas vaincre, Paris, Seuil, 2011, p. 11-12.

7. Mathieu Bélézi, C'était notre terre, Paris, Albin Michel « Le livre de Poche », 2008, p. 350-351.

8. Laurent Mauvignier, Des hommes, Paris, Minuit, 2009/2011, p. 134. 
9. Gerald Prince montre que cette dénomination, pouvant apparaître arbitraire, serait néanmoins en mesure de déterminer un tournant dans les études proposées par les narratologies qui ont cours depuis les années 1980. Voir Gerald Prince, « Narratologie classique et post-classique ", in vox-poetica, http://www.vox-poetica.org/t/articles/ prince.html, consulté le 20 juillet 2016.

10. Notre définition de la « focalisation » est en ce sens très proche de l'acception que lui confère Shlomith Rimmon-Kenan : celle-ci la détermine comme un foyer d'orientation visuelle impliquant des dimensions optiques, psychologiques et idéologiques. Shlomith Rimmon-Kenan, Narrative Fiction : Contemporary Poetics. London/New York, Routledge, 1983, p. 71 et p. 79 à 81.

11. Gerald Prince détermine le « focalisé » comme « l'objet de la focalisation », ce qui est présenté via la perspective du focalisateur, consistant en « le sujet de la focalisation; le porteur du point de vue ; le point focal, qui gouverne la focalisation », en orientant notre lecture. Cf. Gerald Prince, Dictionary of Narratology, Lincoln, University of Nebraska Press, 1987, p. 32 (notre traduction). Sur les enjeux liés à la focalisation, on pourra aussi consulter Mieke Bal, Narratologie. Essais sur la signification narrative dans quatre romans modernes, Paris, Klinksieck, 1977 pour la traduction française, notamment les pages 32 à 38.

12. Dans cette situation narrative, « un même personnage remplit une double fonction : en tant que narrateur (je-narrant) il assume la narration du récit, et en tant qu'acteur (jenarré) il joue un rôle dans l'histoire (personnage-narrateur = personnage-acteur). » Voir Jaap Lintvelt, Essai de typologie narrative - Le " point de vue ». Théorie et analyse, Paris, Librairie José Corti, 1981, p. 38. Nous pourrions ainsi représenter cette perspective par la modélisation suivante : personnage $=$ focalisateur $=$ héros $=$ narrateur.

13. A partir d'une analyse de l'esthétique stendhalienne, George Blin emploie cette expression afin de qualifier la perspective partielle d'un narrateur placé à hauteur de personnage. George Blin, Stendhal et les problèmes du roman, Paris, Librairie José Corti, 1954, p. 115.

14. L'on renvoie à la " perspective par échelonnement » déclinée par Wolfgang Iser, L'acte de lecture. Théorie de l'effet esthétique, Bruxelles, Pierre Mardaga, 1976, 1985, p. 191.

15. Dorrit Cohn propose effectivement une analyse du monologue intérieur dans le roman, suivant une narration à la première ou à la troisième personne du singulier. Cf. Dorrit Cohn, La transparence intérieure. Modes de représentation de la vie psychique dans le roman, Paris, Seuil, 1981 pour la traduction française, p. 30-33.

16. Andrea Del Lungo parle d'incipit « in media verba » pour qualifier ces « seuils » qui immergent de manière intense le lecteur dans l'œuvre. Andrea Del Lungo, L'incipit romanesque, Paris, Seuil, Coll. « Poétique », 2003 pour la traduction française, p. 122. Nous reprenons quant à nous le fréquentatif latin, qui nous semble plus adéquat pour rendre compte de la turbulence de la pensée en mouvement.

17. A travers notre expérience temporelle du quotidien, nous avons la sensation d'enchaîner, de manière enchevêtrée, des épisodes tels des « histoires "non (encore) racontées" ", en train de se faire. Voir Paul Ricœur, Temps et récit. Tome II : La configuration dans le récit de fiction, Paris, Seuil, 1984, p. 143-144.

18. Précisons que l'instance réceptrice, telle que nous l'envisageons, est de l'ordre du « lisant », suivant la tripartition des « régimes de croyance » proposée par Vincent Jouve afin de comprendre les enjeux liés à l'effet-personnage dans le roman. Le " lisant » correspond au lecteur qui, placé dans une « illusion référentielle fragile », accepte le pacte de lecture qui lui est proposé. Les techniques et les procédés narratifs que nous 
avons relevés procèdent d'une réalité textuelle qui n'est peut-être pas incontestable mais qui, tangible, impose au lecteur des contraintes fortes. Cf. Vincent Jouve, L'effet-personnage dans le roman, Paris, PUF, 2001, p. 85-89.

19. Umberto Eco, par exemple, soulève tout l'attachement sentimental qui peut saisir le lecteur vis-à-vis de personnages de fiction. Voir Umberto Eco, «Quelques commentaires sur les personnages de fiction ", SociologieS, Dossiers, Émotions et sentiments, réalité et fiction , juin 2010, http://sociologies.revues.org/3141, consulté le 20 juillet 2016.

20. Pour les références qui suivent, voir Christian Metz, « Le film de fiction et son spectateur ", Communications, 1975, n²3, p. 108-135. Nous faisons référence à l'expression forgée par Christian Metz, quoique ce dernier s'intéresse à la posture spectatorielle du sujet en présence du dispositif cinématographique.

21. Sur ce néologisme, qui renvoie à une forme de « scopophilie ", nous renvoyons à Jean Clair, « La vision de Méduse » in Jean-Bertrand Pontalis (dir.), Le mal, Paris, Gallimard, « Folio essais », 1988, p. 143-163.

22. Le verbe « muser » apparaît pour la première fois dans le roman Perceval de Chrétien de Troyes, au moment où le protagoniste contemple, dans une forme d'envoûtement hypnotique, des gouttes de sang dans la neige.

23. Cf. Monika Fludernik, Towards a "Natural" Narratology, London/New York, Routledge, 1996, p. 6-14.

24. Pour les références qui suivent, nous nous fondons en particulier sur Gilles Deleuze, Présentation de Sacher-Masoch, Le froid et le cruel, Paris, Minuit, 1967 ; Maurice Blanchot, Lautréamont et Sade, Paris, Minuit, 1963 ; Georges Bataille, La littérature et le mal, Paris, Gallimard, 1957, etc.

25. Gilles Deleuze écrit ainsi : « Au lieu de vivre la répétition comme une conduite à l'égard d'un plaisir obtenu ou à venir, au lieu que la répétition soit commandée par l'idée d'un plaisir à retrouver ou à obtenir, voilà que la répétition se déchaîne, est devenue indépendante de tout plaisir préalable. C'est elle qui est devenue idée, idéal. » Voir Gilles Deleuze, op. cit., p. 104.

26. Maurice Merleau-Ponty met ainsi en exergue ce processus chiasmique qui entrelace mutuellement le corps percevant et les objets du monde. Voir Maurice Merleau-Ponty, Le visible et l'invisible suivi de Notes de travail, Paris, Gallimard, « Tel », 1964, en particulier le chapitre « L'entrelacs - le chiasme », p. 174.

27. Le processus d'« hallucination négative » est caractérisé par un « double retournement, dans les échanges qui se reconstituent entre le spectateur et le spectacle, de part et d'autre de la rampe ", ce qui fait de la scène (entendue au sens concret de scène théâtrale mais, au-delà, en un sens évidemment symbolique) un « objet transnarcissique », permettant au sujet de se découvrir dans un rapport d'altérité absolue. Voir André Green, Un ceil en trop - Le complexe d'Ædipe, Paris, Minuit, 1969, en particulier le prologue : «La lecture psychanalytique des tragiques », p. 13. A sa suite, François Richard entreprend l'analyse de ce processus de désubjectivationresubjectivation qui anime le lecteur, dans la perspective ontologique d'un sujet amené à s'appréhender lui-même à travers la « représentation de l'absence de représentation ». Voir François Richard, «Ce que la littérature apprend au psychanalyste », Revue française de psychanalyste, 2009, Vol. 73, p. 165-182.

28. Nous employons le terme de «braconnage » à la suite de Michel de Certeau qui, dans une comparaison poétique entre le marcheur et le liseur, propose de concevoir le lecteur tel un « braconneur » de l'espace métamorphosant, au fil de sa lecture, le texte littéraire. 
Voir Michel de Certeau, L'invention du quotidien. Arts de faire 1, Paris, Gallimard, 1990, «Introduction générale », p. XLIV.

29. Pour les références qui suivent, consulter Julia Kristeva, Pouvoirs de l'horreur : essai sur l'abjection, Paris, Seuil, 1980.

30. Ces deux ordres de sensations sont décrits par Roger Caillois à travers sa typologie des jeux de fiction : la « mimicry » serait de l'ordre du « simulacre », bien délimitée dans ses dimensions spatio-temporelles, tandis que l'« ilinx» (signifiant « le tourbillon d'eau », en grec) tiendrait d'une forme de « vertige ", d'une perte de contrôle tourbillonnant du sujet. Voir Roger Caillois, Les jeux et les hommes - le masque et le vertige, Paris, Gallimard, « Folio/Essais », 1958, 1967, p. 47.

31. Le « double » se rapporte à un « ego alter, que le vivant ressent en lui, à la fois extérieur et intérieur, tout le long de son existence. » Cf. Edgar Morin, L'homme et la mort, Paris, Seuil, Coll. « Points », 1970, p. 153.

32. Voir Sigmund Freud, Totem et tabou - Interprétation par la psychanalyse de la vie sociale des peuples primitifs, Paris, Petite Bibliothèque Payot, 1968, p. 29.

33. La conscience morale apparaît comme la « conscience angoissante » de céder à la tentation $\mathrm{du}$ « tabou » inscrit essentiellement chez l'humain : «C'est la crainte de l'exemple contagieux, de l'impulsion à l'imitation, donc de la nature infectieuse du tabou, qui entre en jeu. » Op. cit., p. 86.

34. Voir Jean Baudrillard, Les stratégies fatales, Paris, Grasset\&Fasquelle, « Biblio Essais », 1983, p. 55-57.

35. On pourrait s'interroger sur le caractère inachevé de ces « histoires potentielles » non encore racontées, qui sont en train d'être racontées, qui ont besoin d'être racontées. Voir Paul Ricœur, op. cit., p. 141-144.

36. Voir Julia Kristeva, op. cit., p. 20-22.

37. Le sujet revivrait ainsi, toujours différente, cette expérience d'une vacillation, d'une oscillation, à travers l'appréhension de « sujets-autres-que-moi » à travers la lecture d'un « espace intermédiaire », comme zone limite que Michel de M’Uzan nomme «spectre d'identité» : il s'agirait d'un espace liminaire, à travers lequel le « je, le Moi-Je se spécifie aléatoirement [...], et en fonction des déplacements de la libido narcissique, tout au long des franges de ce spectre, depuis un pôle interne occupé par la représentation du sujet lui-même jusqu'à un pôle externe qui coïncide avec l'image de l'autre ». Cf. Michel de M'Uzan, Aux confins de l'identité, Paris, Gallimard « Connaissance de l'inconscient », p. 18. 38. La lecture inspirerait littéralement le sujet de ses «flux », de ses « pulsions », de ses « mots d'ordre », par le corps duquel ils passeraient ; mais le lecteur le ferait advenir comme territoire parcouru de tels tremblements. Voir Bertrand Abraham, « À propos de la relecture ", Semen, n¹, 1983, http://semen.revues.org/4251, consulté le 20 juillet 2016. 39. Marielle Macé, Façons de lire, manières d'être, op. cit., p. 101.

40. Le " projet d'autonomie » (qui est davantage autonomisation) du sujet, tel que le conçoit Cornelius Castoriadis, se fonde sur le principe d'un idéal jamais réalisé, correspondant à « la domination du conscient sur l'inconscient ». Il repose effectivement sur l'idée de la construction permanente d'un discours autonome, prenant pour matériau le discours de l'Autre comme une part d'hétéronomie qui s'autonomise non seulement en permanence, mais qui constitue aussi la possibilité de la créativité sans cesse renouvelée du sujet. Voir Cornelius Castoriadis, L'institution imaginaire de la société, Paris, Seuil, 1975, p. 155. 


\section{ABSTRACTS}

A partir d'extraits de romans de la littérature française du XXIe siècle relatant la guerre d'Algérie, nous nous proposons de faire émerger deux degrés d'oscillation identitaire du sujet amené à se projeter dans la pensée de personnages de fiction singuliers, à la faveur de techniques et de procédés narratifs. Nous nous concentrons ainsi sur une expérience de la lecture de romans - romans entendus comme des "dispositifs", des « espaces transitionnels » fondamentalement anthropomorphiques - susceptible de faire émerger une ontologie du lecteur tendue vers l'idée d'autonomisation.

INDEX

Mots-clés: Réception ; Lecture ; Dispositif ; Identité ; Altération

Geographical index: France ; Algérie

\section{AUTHOR}

\section{HÉLÈNE CROMBET}

Docteur en Sciences de l'Information et de la Communication Membre associée de l'EA 4426 MICA Attachée Temporaire d'Enseignement et de Recherche U.F.R. S.T.C. Université Bordeaux Montaigne 